BMJ

Open

Gastroenterology

\title{
Assessment of health utilities and quality of life in patients with non-alcoholic fatty liver disease
}

\author{
Mehmet Sayiner, ${ }^{1}$ Maria Stepanova, ${ }^{2}$ Huong Pham, ${ }^{1}$ Bashir Noor, ${ }^{1}$ \\ Mercedes Walters, ${ }^{2}$ Zobair M Younossi ${ }^{1,2}$
}

To cite: Sayiner M,

Stepanova M, Pham H, et al. Assessment of health utilities and quality of life in patients with non-alcoholic fatty liver disease. BMJ Open Gastro 2016;3:e000106

doi:10.1136/bmjgast-2016000106

Received 9 June 2016 Revised 25 July 2016 Accepted 26 July 2016

\section{${ }^{1}$ Betty and Guy Beatty Center for Integrated Research, Inova Health System, Falls Church, Virginia, USA ${ }^{2}$ Department of Medicine, Center for Liver Diseases, Inova Fairfax Hospital, Falls Church, Virginia, USA}

Correspondence to Professor Zobair M Younossi;

zobair.younossi@inova.org

\section{ABSTRACT}

Background: Non-alcoholic fatty liver disease (NAFLD) is one of the most common causes of chronic liver disease associated with increased liverrelated mortality. Additionally, NAFLD could potentially impair health-related quality of life. Although an approved treatment for NAFLD does not exist, a number of new drugs for treatment of NAFLD are being developed. As the efficacy and safety of these regimens are being established, their cost-effectiveness, which requires the use of quality of life metrics and health utility scores to quality-adjusted outcomes, must also be assessed. The aim of this study was to report quality of life and health utilities in patients with NAFLD with and without cirrhosis for future use.

Methods: Patients with NAFLD were seen in an outpatient clinic setting. Each patient had extensive clinical data and completed the Short Form-36 (SF-36 V.1) questionnaire. The SF-6D health utility scores were calculated.

Results: There were 89 patients with the spectrum of NAFLD completed the SF-36 questionnaire: 59 with non-cirrhotic NAFLD and 30 with cirrhosis. Patients with NAFLD had significantly lower quality of life and health utility scores than the general population (all $p<0.0001)$. Furthermore, patients with cirrhosis had lower quality of life and utility scores than non-cirrhotic NAFLD patients: SF-6D $0.660 \pm 0.107$ in non-cirrhotic NAFLD vs $0.551 \pm 0.138$ in cirrhotic NAFLD $(p=0.0003)$. Conclusions: Health utilities and quality of life scores are impaired in patients with cirrhotic NAFLD. These values should be used in cost-effectiveness analysis of the upcoming treatment regimens for advanced NAFLD.

\section{INTRODUCTION}

Non-alcoholic fatty liver disease (NAFLD) is a spectrum of liver disease, ranging from simple steatosis to cirrhosis and its complications such as hepatocellular carcinoma. ${ }^{1-3}$ In 2016, NAFLD is considered as one of the most prevalent forms of chronic liver disease in the world. ${ }^{4-5}$ Although NAFLD is mostly asymptomatic, patients may experience

\section{Summary box}

What is already known about this subject?

- NAFLD is one of the most prevalent forms of chronic liver disease globally, with an increasing prevalence.

- NAFLD is often asymptomatic, but patients can experience fatigue, decreased activity, and emotional health impairment.

- NAFLD is an umbrella term, and some forms of fatty liver disease can progress to advanced stages.

- NAFLD is also associated with tremendous economic burden.

What are the new findings?

- Compared to the general population, patients with NAFLD had lower health-related quality-of-life (HRQL) and health utility scores.

- Even in the absence of cirrhosis, patients with NAFLD had lower HRQL scores.

- The presence of advanced stages of liver disease increases the reduction in HRQL and health utility scores.

How might it impact on clinical practice in the foreseeable future?

- The degree of fibrosis and advanced stages of liver disease will affect patients' HRQL and health utility scores.

- Treatment of NAFLD or even the prevention of disease progression can decrease the reduction in $\mathrm{HRQL}$ and health utility scores.

fatigue, decreased activity, and emotional health impairment, which can impact their health-related quality of life (HRQL) ${ }^{5-8}$

In addition to its clinical outcomes and patients' experience outcomes, NAFLD is known to be associated with a tremendous economic burden. ${ }^{8-11}$ In this context, quality of life adjustment is usually performed using health utilities which are based on patients' preference for a health status. ${ }^{12}$ In particular, policymakers typically rely on economic analyses which often involve calculation of quality-adjusted life years (QALYs) and other 
quality-adjusted outcomes. The QALYs provide a measurement for the years of life gained or lost not only in terms of their quantity but also the quality of these years of life. ${ }^{13} 14$

There are a number of ways to measure health utilities. ${ }^{15} 16$ One popular indirect method to estimate health utilities is to use the SF-6D scores which could be derived from the widely validated and used quality of life questionnaire, the Medical Outcome Study-Short Form 36 (SF-36). ${ }^{17}{ }^{18}$ Given the extent of the economic burden of NAFLD, we believe that deriving health utility scores in patients with NAFLD will be useful for future economic analyses of all upcoming treatments. Therefore, our aim was to assess HRQL and SF-6D health utility scores of patients with NAFLD with and without NAFLD-related cirrhosis.

\section{METHODS}

Patients with chronic liver disease for whom clinical and quality of life data had been collected for our quality of life database were considered for this study. We included only patients with an established histological diagnosis of NAFLD with or without cirrhosis who provided an informed consent; the presence of cirrhosis was determined from liver biopsies. The following patients were excluded from the study population: patients with viral hepatitis, with significant alcohol intake $(>20 \mathrm{~g}$ /day for men, $>10 \mathrm{~g} /$ day for women), and with other causes of chronic liver disease. Also, for this study, only patients with a completed SF-36 questionnaire were selected.

\section{Health-related quality of life}

The SF-36 questionnaire (Version 1) includes eight individual $\mathrm{HRQL}$ domains:

- Physical functioning (PF) domain reflects how much a patient's physical activities are limited because of their health.

- Role physical (RP) domain reflects how much the patient's physical health impacts their work and daily activities.

- Bodily pain (BP) domain evaluates the patient's limitations because of pain.

- General health $(\mathrm{GH})$ domain measures how a patient sees their personal health and the potential for decline.

- Vitality (VT) domain reflects how tired/full of energy the patient feels.

- Social functioning (SF) domain measures how much the patient's physical or emotional problems interfere with their normal social activities.

- Role emotional (RE) domain assesses the impact of the patient's emotional problems on their work and daily activities.

- Mental health (MH) domain reflects the patient's state of emotional feeling (eg, nervous, peaceful, happy).

For calculation of all these domains, patients' responses (all on Likert scales of various sizes, from 2 to
6) were averaged and then transformed to range from 0 to 100 with higher scores representing better health. Then, these domain scores were normalised to the 1994 US population means and SDs, and used for calculation of the two summary scores:

- Physical Component (Summary) Score (PCS) summarises the physical health-related items of SF-36: PF, $\mathrm{RP}, \mathrm{BP}$, and GH.

- Mental Component (Summary) Score (MCS) summarises the MH-related items of SF-36: VT, SF, RE, and $\mathrm{MH}$.

The summary scores were calculated from the normalised domain scores using weights reported from factor analysis of the SF-36 items and with adjustment for interitem correlations. ${ }^{19}$ These scores were then transformed to have a mean of 50 and an SD of 10.

Using the SF-36 questionnaire, we also calculated the SF-6D health utility score. The rationale for the need of this metric comes from the fact that the SF-36 instrument does not explicitly incorporate patients' preferences into its scoring algorithm. This makes the SF-36 domains unsuitable for direct use in economic analyses which require health utility scores for calculation of qualityadjusted outcomes. On the other hand, calculation of the SF-6D metric includes applying a 'preference tariff' to patients' responses, which reflects how 'valued' a particular state of health would be to a patient. The most recent non-parametric Bayesian algorithm was used for calculation of the SF-6D scores from the SF-36 items. ${ }^{20}$

\section{Statistical analysis}

We compared all collected demographic and clinical parameters, together with all of the SF-36 domains, SF-36 summary scores, and SF-6D health utility scores, between patients with cirrhotic NAFLD and noncirrhotic NAFLD. A non-parametric Mann-Whitney test was used to compare continuous and pseudo-continuous parameters (such as age or HRQL scores), and a $\chi^{2}$ test was used for categorical parameters (such as gender). For all tests, $\mathrm{p}$ values not exceeding the 0.05 threshold were considered statistically significant.

All analyses were performed using SAS software (SAS, Cary, North Carolina, USA). The study was approved by Institutional Review Board.

\section{RESULTS}

A total of 89 patients with NAFLD who completed SF-36 questionnaire were used in this study. Of those, 59 had non-cirrhotic NAFLD (age: $49.1 \pm 10.4$, \% male: $37.3 \%$, BMI: 39.9 \pm 8.8 ), and 30 had NAFLD-related cirrhosis (age: $54.1 \pm 10.8$, \% male: $56.7 \%$ ). Patients with noncirrhotic NAFLD were younger in comparison to patients with cirrhotic NAFLD $(p<0.05)$. The gender and race distribution was similar between the two groups (all $\mathrm{p}>0.05$ ) (table 1). Of patients with complete clinical data, $45.8 \%$ and $13.8 \%$ had type 2 diabetes and cardiovascular disease, respectively. Of patients with cirrhosis, 
Table 1 Comparison of the HRQL scores and health utilities between patients with and without NAFLD-related cirrhosis

\begin{tabular}{|c|c|c|c|c|}
\hline & Cirrhotic NAFLD & Non-cirrhotic NAFLD & p Value & All NAFLD \\
\hline $\mathrm{N}$ & 30 & 59 & & 89 \\
\hline Age, years & $54.1 \pm 10.8$ & $49.1 \pm 10.4$ & 0.0462 & $50.8 \pm 10.7$ \\
\hline Male gender & $17(56.7 \%)$ & $22(37.3 \%)$ & 0.08 & $39(43.8 \%)$ \\
\hline \multicolumn{5}{|l|}{ Race } \\
\hline Caucasian & $20(83.3 \%)$ & $43(72.9 \%)$ & 0.31 & $63(75.9 \%)$ \\
\hline African-American & $2(8.3 \%)$ & $6(10.2 \%)$ & 0.80 & $8(9.6 \%)$ \\
\hline Hispanic & $1(4.2 \%)$ & $4(6.8 \%)$ & 0.65 & $5(6.0 \%)$ \\
\hline Asian & $1(4.2 \%)$ & $4(6.8 \%)$ & 0.65 & $5(6.0 \%)$ \\
\hline \multicolumn{5}{|l|}{ HRQL and health utilities } \\
\hline $\mathrm{PF}(84.5)^{*}$ & $37.5 \pm 30.8 \dagger$ & $72.0 \pm 22.0 \dagger$ & $<0.0001$ & $60.4 \pm 30.0 \dagger$ \\
\hline $\operatorname{RP}(81.2)^{*}$ & $21.7 \pm 35.8 \dagger$ & $57.5 \pm 40.8 \dagger$ & 0.0001 & $45.4 \pm 42.5 \dagger$ \\
\hline $\mathrm{BP}(75.5)^{*}$ & $53.1 \pm 30.7 \dagger$ & $66.9 \pm 23.8 \dagger$ & 0.0365 & $62.2 \pm 26.9 \dagger$ \\
\hline $\mathrm{GH}(72.2)^{*}$ & $34.1 \pm 23.7 \dagger$ & $58.5 \pm 18.1 \dagger$ & $<0.0001$ & $50.3 \pm 23.1 \dagger$ \\
\hline VT $(61.1)^{*}$ & $29.8 \pm 24.2 \dagger$ & $46.8 \pm 19.8 \dagger$ & 0.0016 & $41.1 \pm 22.8 \dagger$ \\
\hline SF $(83.6)^{*}$ & $50.0 \pm 32.7 \dagger$ & $78.4 \pm 22.5$ & 0.0001 & $68.8 \pm 29.4 \dagger$ \\
\hline $\operatorname{RE}(81.3)^{\star}$ & $38.9 \pm 45.6 \dagger$ & $73.4 \pm 37.0$ & 0.0006 & $61.8 \pm 43.1 \dagger$ \\
\hline $\mathrm{MH}(74.8)^{*}$ & $67.3 \pm 18.4 \dagger$ & $75.8 \pm 17.7$ & 0.0512 & $72.9 \pm 18.3$ \\
\hline Physical Component Summary (50)* & $30.4 \pm 11.3 \dagger$ & $42.5 \pm 9.8 \dagger$ & $<0.0001$ & $38.5 \pm 11.8 \dagger$ \\
\hline Mental Component Summary $(50)^{\star}$ & $43.0 \pm 11.5 \dagger$ & $49.6 \pm 10.3$ & 0.0068 & $47.3 \pm 11.1 \dagger$ \\
\hline SF-6D health utility $(0.78)^{\star}$ & $0.551 \pm 0.138 \dagger$ & $0.660 \pm 0.107 \dagger$ & 0.0003 & $0.623 \pm 0.129 \dagger$ \\
\hline
\end{tabular}

${ }^{*}$ General population mean. ${ }^{21} 22$

$\dagger p<0.05$ when compared to the general population mean.

$\mathrm{BP}$, bodily pain; GH, general health; HRQL, health-related quality of life; MH, mental health; NA, not available for the cohort; PF, physical functioning; RE, role emotional; RP, role physical; SF, social functioning; VT, vitality.

22/30 (73.3\%) were Child-Turcotte-Pugh (CTP) class A and 8/30 (26.7\%) were CTP class B or C.

As shown in table 1 , all HRQL scores were significantly lower in patients with cirrhotic NAFLD (all $p \leq 0.05$ ). In particular, the utility scores in the two groups were as follows: $0.660 \pm 0.107$ in non-cirrhotic NAFLD vs 0.551 \pm 0.138 in cirrhotic NAFLD ( $p=0.0003$ ). Despite this, even in non-cirrhotic patients, most of their HRQL scores, including all of the domain scores related to patients' physical health and also patients' health utility scores, were significantly lower in comparison to the general US population means (all $\mathrm{p}<0.001)^{2122}$ (figure 1 ).

In multiple regression analysis, after adjustment for age, gender and ethnicity, having cirrhosis was independently associated with lower HRQL and utility scores in patients with NAFLD. In particular, the $\beta$ values were as follows: $-32.6 \pm 6.2 \quad(\mathrm{p}<0.0001)$ for $\mathrm{PF},-34.9 \pm 10.1$ $(p=0.0009)$ for RP, $-13.0 \pm 6.4 \quad(p=0.0467)$ for $B P,-24.0$ $\pm 5.0(\mathrm{p}<0.0001)$ for $\mathrm{GH},-15.5 \pm 5.3(\mathrm{p}=0.0049)$ for $\mathrm{VT}$, $-28.4 \pm 6.6(\mathrm{p}<0.0001)$ for $\mathrm{SF},-34.4 \pm 10.3(\mathrm{p}=0.0012)$ for $\mathrm{RE}$, not significant for $\mathrm{MH} \quad(\mathrm{p}=0.17), \quad-11.7 \pm 2.5$ $(\mathrm{p}<0.0001)$ for PCS, $-6.1 \pm 2.7(\mathrm{p}=0.0267)$ for MCS, and $-0.102 \pm 0.030 \quad(p=0.0009)$ for SF-6D utilities. There were no other independent predictors of HRQL metrics and utilities in patients with NAFLD that would reach statistical significance (all $\mathrm{p}>0.05$ ). In particular, no independent associations with HRQL scores were found for BMI, history of CVD, and type 2 diabetes (all $p>0.10$ ). In patients with cirrhosis, we similarly did not find any association of HRQL score with the CTP class, probably due to limited sample size.

\section{DISCUSSION}

In this study, we assessed the impact of NAFLD on patients' HRQL and health utility scores. Our data clearly show that nearly all HRQL indicators and also health utilities are significantly lower in patients with NAFLD in comparison to the general population. Furthermore, we have shown that more advanced fatty liver disease, as documented by the presence of cirrhosis, had an additional negative impact on patients' HRQL and health utility scores.

NAFLD has increasingly been recognised as one of the most common causes of chronic liver disease worldwide. The shared pathophysiological mechanisms have established strong linkage between NAFLD and obesity and metabolic syndrome. ${ }^{23}$ Besides its substantial epidemiological ${ }^{24}$ and economic ${ }^{1125}$ impacts, NAFLD also causes a significant deterioration of patients' HRQL. Our study revealed that, compared to the general population, patients with NAFLD had lower HRQL and health utility scores, even in the absence of cirrhosis. These findings were in agreement with previous publications in the literature. ${ }^{5} 2627$ In a very recent study among the National Health and Nutrition Examination Survey (NHANES) population, it was found that almost one-fourth of patients with NAFLD reported their health as poor, which was significantly greater than in healthy people. ${ }^{5}$ It was also emphasised that patients with NAFLD had more impairment of their physical health than their MH. Our findings supported these data as for physical component domains, patients with cirrhotic and non-cirrhotic NAFLD had significantly 


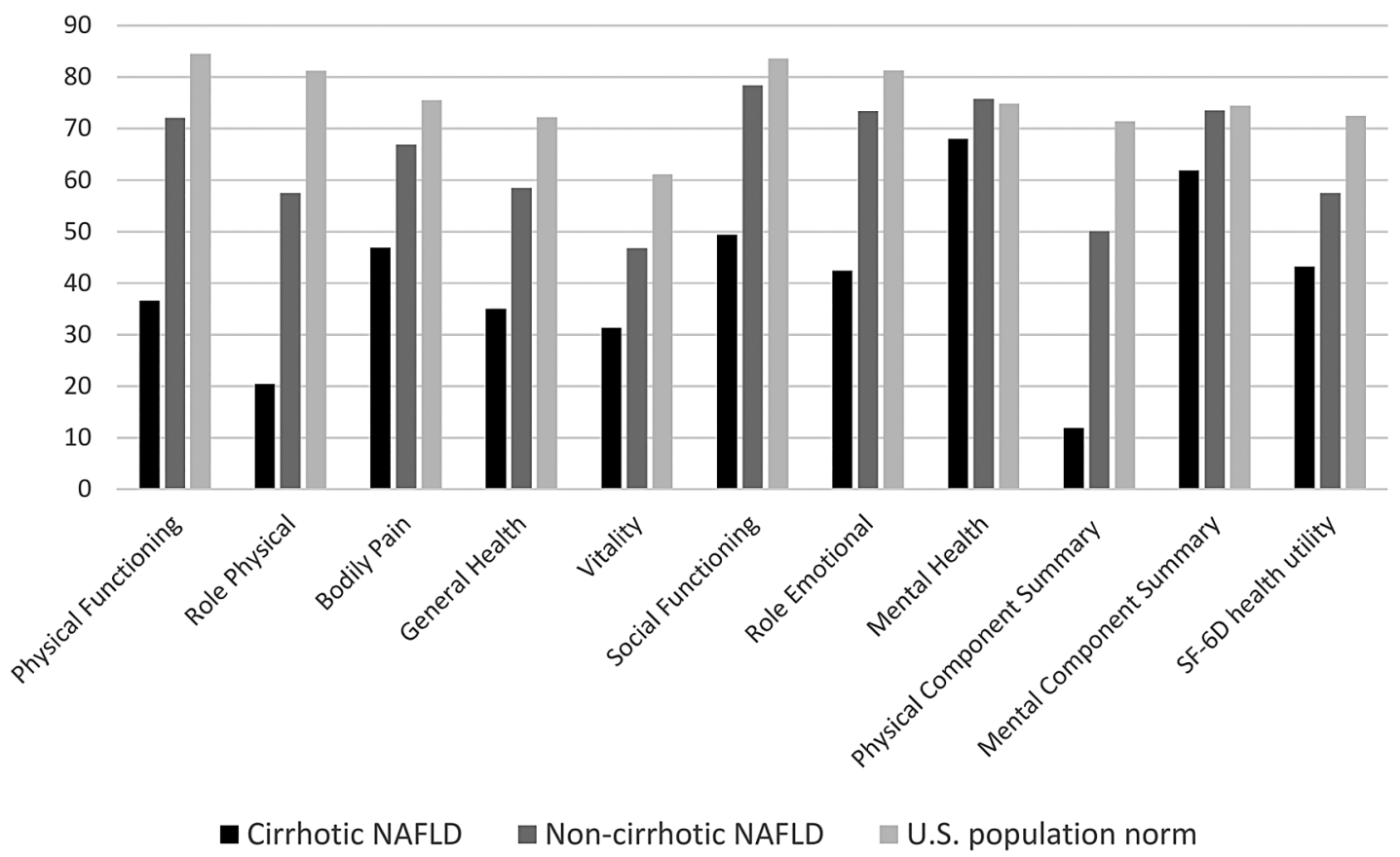

Figure 1 The SF-36 HRQL scores and SF-6D health utilities in patients with cirrhotic and non-cirrhotic NAFLD. Note: The summary scores and the utility scores were transformed to a 0-100 scale for the purpose of presentation. All $p<0.05$, except for MH. HRQL, health-related quality of life; MH, mental health; NAFLD, non-alcoholic fatty liver disease; SF-36, Short Form-36.

lower scores than the general population, whereas for mental component domains, only the scores of cirrhotic patients achieved significance. In a study of Dan et al, HRQL of patients with chronic liver disease caused by different aetiologies was assessed. It was reported that the overall HRQL scores of patients with NAFLD were lower than those of patients with hepatitis B (HBV) and hepatitis C (HCV) infections, and NAFLD was an independent predictor of poorer HRQL. ${ }^{28}$

The impact of disease severity on HRQL was another finding of our study, which was parallel to the findings of previous publications, ${ }^{262729}$ as patients with cirrhotic NAFLD had significantly lower HRQL and health utility scores than patients with non-cirrhotic NAFLD. In a study of David et al, ${ }^{26}$ HRQL of patients with NAFLD was assessed using SF-36. More than $60 \%$ of the study population had non-alcoholic steatohepatitis (NASH), half of which had bridging fibrosis or cirrhosis. It was found that patients who had progressed to NASH had lower HRQL scores than patients with simple steatosis, and cirrhotic patients had the least HRQL scores. In another study, Afendy et $a l^{27}$ investigated the HRQL of patients with chronic liver disease and compared the findings of cirrhotic patients according to their Child-Pugh class. It was found that patients with Child-Pugh class A cirrhosis had significantly higher scores on all SF-36 domains than patients with Child-Pugh class B and C cirrhosis, and there was no significant difference between the latter two classes. In our study, patients with cirrhosis had significantly lower HRQL and health utility scores than patients without cirrhosis and the general population.
The limitations of this study include a limited sample size, limited clinical data, and the absence of a better granularity of the NAFLD spectrum (such as the stages of non-cirrhotic steatohepatitis or decompensated cirrhosis). Also, the retrospective analysis does represent a limitation of the study design, and future prospective studies are warranted. Nevertheless, calculating SF-6D scores directly from the SF-36 instrument can provide the health utility scores that can be used for all future economic analyses related to NAFLD. To the best of our knowledge, this information is currently not available.

In conclusion, this study clearly showed that compared to the general population, patients with NAFLD had significantly lower HRQL and health utility scores, which worsen with the advanced stages of the disease. We believe that having these data is extremely important in the context of NAFLD becoming increasingly recognised as the most important chronic liver disease worldwide.

Acknowledgements The authors thank Pegah Golabi, MD; Deena Hallaji; Manirath Srishord and Brian Lam, PA-C for their great support during the study process.

Contributors MSa and MSt participated in the study design and helped in interpretation of the data and to draft the manuscript. MSt performed the statistical analysis and helped in interpretation of the data. HP, BN, and MW made contributions to the study design and interpretation of the data. ZMY conceived of the study, participated substantially in its design and coordination, and helped to draft the manuscript. Each author reviewed the final version of the manuscript and approved it for publication.

Funding This study was internally funded.

Competing interests ZMY is a consultant to BMS, Gilead, AbbVie, Intercept, GSK, and Salix.

Ethics approval The study was approved by Inova Institutional Review Board. 
Provenance and peer review Not commissioned; externally peer reviewed.

Data sharing statement No additional data are available.

Open Access This is an Open Access article distributed in accordance with the Creative Commons Attribution Non Commercial (CC BY-NC 4.0) license, which permits others to distribute, remix, adapt, build upon this work noncommercially, and license their derivative works on different terms, provided the original work is properly cited and the use is non-commercial. See: http:// creativecommons.org/licenses/by-nc/4.0/

\section{REFERENCES}

1. Fazel $Y$, Koenig $A B$, Sayiner $M$, et al. Epidemiology and natural history of non-alcoholic fatty liver disease. Metabolism 2016;65:1017-25.

2. Golabi P, Sayiner M, Fazel Y, et al. Current complications and challenges in nonalcoholic steatohepatitis screening and diagnosis. Expert Rev Gastroenterol Hepatol 2015;10:63-71.

3. Chalasani N, Younossi Z, Lavine JE, et al. The diagnosis and management of non-alcoholic fatty liver disease: practice Guideline by the American Association for the Study of Liver Diseases, American College of Gastroenterology, and the American Gastroenterological Association. Hepatology 2012;55:2005-23.

4. Younossi ZM, Koenig AB, Abdelatif D, et al. Global epidemiology of non-alcoholic fatty liver disease-meta-analytic assessment of prevalence, incidence and outcomes. Hepatology 2016;64: 73-84.

5. Golabi P, Otgonsuren M, Cable R, et al. Non-alcoholic fatty liver disease (NAFLD) is associated with impairment of health-related quality of life (HRQOL). Health Qual Life Outcomes 2016;14:18.

6. Loria A, Escheik C, Gerber NL, et al. Quality of life in cirrhosis. Curr Gastroenterol Rep 2013;15:301.

7. Younossi Z, Henry L. Contribution of alcoholic and non-alcoholic fatty liver disease to the burden of liver-related morbidity and mortality. Gastroenterology 2016;150:1778-85.

8. Younossi ZM, Henry L. Economic and quality-of-life implications of non-alcoholic fatty liver disease. Pharmacoeconomics 2015;33:1245-53.

9. Younossi ZM, Zheng L, Stepanova M, et al. Trends in outpatient resource utilizations and outcomes for Medicare beneficiaries with nonalcoholic fatty liver disease. J Clin Gastroenterol 2015;49:222-7.

10. Ghamar Chehreh ME, Vahedi M, Pourhoseingholi MA, et al. Estimation of diagnosis and treatment costs of non-alcoholic fatty liver disease: a two-year observation. Hepat Mon 2013;13:e7382.

11. Baumeister SE, Volzke $\mathrm{H}$, Marschall $\mathrm{P}$, et al. Impact of fatty liver disease on health care utilization and costs in a general population: a 5-year observation. Gastroenterology 2008;134:85-94.

12. Neumann PJ, Goldie SJ, Weinstein MC. Preference-based measures in economic evaluation in health care. Annu Rev Public Health 2000;21:587-611.

13. Sassi F. Calculating QALYs, comparing QALY and DALY calculations. Health Policy Plan 2006;21:402-8.
14. Siegel JE, Weinstein MC, Russell LB, et al. Recommendations for reporting cost-effectiveness analyses. Panel on Cost-Effectiveness in Health and Medicine. JAMA 1996;276:1339-41.

15. Richardson J, lezzi A, Khan MA, et al. Measuring the sensitivity and construct validity of 6 utility instruments in 7 disease areas. Med Decis Making 2016;36:147-59.

16. Busija L, Pausenberger E, Haines TP, et al. Adult measures of general health and health-related quality of life: Medical Outcomes Study Short Form 36-Item (SF-36) and Short Form 12-Item (SF-12) Health Surveys, Nottingham Health Profile (NHP), Sickness Impact Profile (SIP), Medical Outcomes Study Short Form 6D (SF-6D), Health Utilities Index Mark 3 (HUI3), Quality of Well-Being Scale (QWB), and Assessment of Quality of Life (AQoL). Arthritis Care Res (Hoboken) 2011;63(Suppl 11):S383-412.

17. Stepanova M, Nader F, Cure S, et al. Patients' preferences and health utility assessment with SF-6D and EQ-5D in patients with chronic hepatitis $\mathrm{C}$ treated with sofosbuvir regimens. Aliment Pharmacol Ther 2014;40:676-85

18. Kharroubi SA, Brazier JE, Roberts J, et al. Modelling SF-6D health state preference data using a nonparametric Bayesian method. $J$ Health Econ 2007;26:597-612.

19. Ware JE Jr, Gandek B. Overview of the SF-36 Health Survey and the International Quality of Life Assessment (IQOLA) Project. J Clin Epidemiol 1998;51:903-12.

20. Ara R, Brazier J. Predicting the short form-6D preference-based index using the eight mean short form-36 health dimension scores: estimating preference-based health-related utilities when patient level data are not available. Value Health 2009;12:346-53.

21. Ware J, Snow K, Kosinski M, et al. SF-36 health survey manual and interpretation guide. Boston, MA: New England Medical Center, The Health Institute, 1993.

22. Fryback DG, Dunham NC, Palta M, et al. US norms for six generic health-related quality-of-life indexes from the National Health Measurement study. Med Care 2007;45:1162-70.

23. Mishra A, Younossi ZM. Epidemiology and natural history of non-alcoholic fatty liver disease. J Clin Exp Hepatol 2012;2:135-44.

24. Everhart JE, Ruhl CE. Burden of digestive diseases in the United States Part III: liver, biliary tract, and pancreas. Gastroenterology 2009;136:1134-44.

25. Younossi ZM, Zheng L, Stepanova M, et al. Clinical outcomes and resource utilisation in Medicare patients with chronic liver disease: a historical cohort study. BMJ Open 2014;4:e004318.

26. David K, Kowdley KV, Unalp A, et al. Quality of life in adults with nonalcoholic fatty liver disease: baseline data from the nonalcoholic steatohepatitis clinical research network. Hepatology 2009:49:1904-12.

27. Afendy A, Kallman JB, Stepanova M, et al. Predictors of health-related quality of life in patients with chronic liver disease. Aliment Pharmacol Ther 2009;30:469-76.

28. Dan AA, Kallman JB, Wheeler A, et al. Health-related quality of life in patients with non-alcoholic fatty liver disease. Aliment Pharmacol Ther 2007;26:815-20.

29. Younossi ZM, Boparai N, Price LL, et al. Health-related quality of life in chronic liver disease: the impact of type and severity of disease. Am J Gastroenterol 2001;96:2199-205. 